\title{
Missing Doublet Multiplet as the Origin of the Doublet-Triplet Splitting in SUSY SU(6)
}

\author{
I. Gogoladze A. Kobakhidze and Z. Tavartkiladze \\ Institute of Physics of Georgian Academy of Sciences, Tamarashvili 6, Tbilisi 380077, \\ Republic of Georgia
}

\begin{abstract}
In a $S U(6)$ gauge theory we found the irreducible representation (175-plet) which does not contain the Higgs doublet. Using this representation we construct two SUSY $S U(6)$ models in which the doublet-triplet splitting occurs naturally, without fine tuning. The crucial role is played by the "custodial" global $S U(2)_{H}$ in combination with discrete or continuous $R$ symmetries.
\end{abstract}

\section{Introduction}

The problem of gauge hierarchy is concomitant to any Grand Unified Theories (GUT). While solving this problem two questions must be understood: 1 . Why the large scale of GUT $\left(M_{G} \sim 10^{16} \mathrm{GeV}\right)$ is not felt by the electroweak scale $m_{W} \sim 100 \mathrm{GeV}$ ? In other words, what is the reason that $S U(2)_{W} \times U(1)_{Y}$ symmetry breaking scale is so small and stable against radiative corrections? 2. How does the Higgs doublets remain light when their color triplet partners from the same irreducible representation (IRREP) must be superheavy in order to avoid fast proton decay? The latter problem is known as the doublettriplet (DT) splitting problem.

The first problem is solved by supersymmetry, which can render the weak scale to be stable against radiative corrections. In SUSY GUTs the so called "technical" solution [1] of the second problem is based on 'fine tuning': after GUT gauge $G$ group breaking to $G_{S M}=S U(3)_{C} \times S U(2)_{W} \times U(1)_{Y}$, by proper choice of the tree level potential parameters, the mass of the doublet component may be imposed to be zero (or of order $100 \mathrm{GeV}$ ) while the mass of triplet fragment is order $M_{G}$. Unfortunately this looks very unnatural.

The actual task is to obtain DT splitting without fine tuning. Several possibilities were suggested in the literature:

a) In "sliding singlet" model [2] the DT splitting occurs in exact SUSY limit, but after SUSY breaking the hierarchy is spoiled [3].

b) Missing partner mechanism [4 is based on purely group-theoretical arguments and it can be implemented in SUSY $S U(5)$ theory. By introducing the missing doublet multiplets: $75+50+\overline{50}$ and by employing several symmetries one can exclude the direct mass term for the Higgs superfields $5+\overline{5}$, 
containing the Higgs doublets. Consequently these doublets remain massless while their triplet partners acquire large $\left(\sim M_{G}\right)$ masses by the mixing with the massive triplets from $50+\overline{50}$. However, besides the unpleasant fact that this mechanism employs huge representations, it can be spoiled by the possible nonrenormalizable terms which are permitted by all symmetries.

c) Missing VEV mechanism [5] closely resembles the missing partner mechanism and it can be realized in SUSY $S O(10)$ within the basic multiplets: $45+10+10^{\prime}$. If 45 has VEV in B-L direction, then the $10 \cdot 45 \cdot 10^{\prime}$ term in the superpotential renders the Higgs doublets massless, while the triplets acquire the large masses $\sim M_{G}$. These solutions also suffer instability against the higher order terms. It was shown [6] that DW mechanism can be protected against the effects of higher-dimension operators, but very complicated field content is required.

d) The Goldstone boson mechanism [7, 8, 9] in which the Higgs doublets are identified with the pseudoGoldstone bosons (PGB) of the spontaneously broken global pseudosymmetry looks very promising. The first attempts were done in SUSY $S U(5)$ with the superpotential having the larger $S U(6)$ global symmetry [7]. Implementing this idea in a more consistent way, the models were built based on the $S U(6)$ gauge group [8, 9]. In these models Higgs doublets emerge as the PGB modes due to accidental global $S U(6) \times S U(6)$ pseudosymmetry of the Higgs superpotential. It was shown in ref. [9] that in fact the $S U(6)$ SUSY GUT (or maybe its trivial unitary extensions to $S U(6+N)$ ) are only viable possibilities for the Goldstone boson mechanism. In the same paper by introducing additional discrete symmetries there was constructed models in which higher order terms are harmless and can not spoil the hierarchy.

e) The "custodial" symmetry mechanism [10] also requires the $S U(6)$ gauge group. The Higgs doublet is light since it is related by the custodial symmetry to another doublet which after GUT symmetry breaking becomes an unphysical Higgs. If direct mass term for the 35-adjoint representation is omitted the superpotential is flat and the mass term of the doublets is exactly canceled in the exact SUSY limit. After SUSY breaking due to soft terms cancelation is partial and doublets acquire the masses of order $m_{3 / 2}$. Because of this the intermediate scale is arising in the theory. In general nonrenormalizable terms also may destroy the hierarchy.

In this paper we suggest the natural mechanism for the DT splitting in SUSY $S U(6)$ theory. This mechanism actually lies towards the lines of "custodial" symmetry mechanism 10, but resembles the missing partner mechanism because of peculiarity of the scalar content which we consider. For the $S U(6)$ symmetry breaking the 175 representation is used which does not contain the electroweak doublet fragments. Two models are constructed in which crucial role in DT splitting is played by discrete or continuous $R$ symmetries in such a way as all dangerous higher order operators are excluded from the superpotential. 


\section{General idea: 175-plet and its properties}

$S U(6)$ is the minimal semi-simple group whose adjoint representation contains $G_{S M}$ Higgs doublets. subgroup has the form $35=1+5+\overline{5}+24$, where a pair of doublet-antidoublet is contained in 5 and $\overline{5}$ respectively. When the $S U(6)$ symmetry breaks down to $G_{S M}$ spontaneously, then one pair of doublets from the scalar fields emerge as goldstone bosons eaten by the corresponding gauge fields of $S U(6)$ which become massive through the Higgs mechanism. If there exists another pair of the Higgs doublets in the theory which after the $S U(6)$ breaking remain massless due to some mechanism [8, 9, 10] while the color triplets acquire masses of order $M_{G}$, then in the effective low energy theory we will have one pair of light Higgs doublets as in the minimal supersymmetric standard model.

In a number of $S U(6)$ models the $35=\Sigma_{B}^{A}$ adjoint IRREP was used for the symmetry breaking $\mathbb{8}, 9$, 10]. In exact SUSY limit the potential of 35-plet in general has three degenerate minima corresponding to three following channels of the $S U(6)$ symmetry breaking:

$$
\begin{array}{ll}
\text { a) } & S U(5) \times U(1) \\
\text { b) } & S U(4) \times S U(2) \times U(1) \\
\text { c) } & S U(3) \times S U(3) \times U(1)
\end{array}
$$

In $(1, a)$ and $(1, b)$ cases the $5+\overline{5}$ and $(4, \overline{2})+(\overline{4}, 2)$ fragments from $\Sigma$ are absorbed by the appropriate gauge fields. Both this fragments contain the pair of $G_{S M}$ doublets.

Here we suggest a possibility to break the $S U(6)$ symmetry by the selfconjugate IRREP -175 : $175 \equiv \Phi_{A^{\prime} B^{\prime} C^{\prime}}^{A B C}(A, B, \ldots$ denote the $S U(6)$ indices $)$ which is full antisymmetric with respect to up and down indices and $\sum \Phi_{A B^{\prime} C^{\prime}}^{A B C}=0$.

The decomposition of the 175-plet in terms of the $S U(5)$ subgroup has the following form:

$$
175=75+50+\overline{50}
$$

Neither 75 nor $50(\overline{50})$ contains the Higgs doublet. It is interesting to note that $75+50+\overline{50}$ are just these IRREPs, which were needed for the "missing partner" mechanism [1 to be operative in SUSY SU(5) model; as we see by extending the $S U(5)$ group to $S U(6)$, it is possible to put these IRREPs exactly in the one $S U(6)$ IRREP.

175 cannot break $S U(6)$ group into the $(1, a)$ and $(1, b)$ channels, so that only the $(1, c)$ channel is possible. With respect to the $S U(3) \times S U(3) \times U(1)$ subgroup 175 decomposes into:

$$
\begin{gathered}
175=(1,1)_{0}+(1,1)_{-6}+(1,1)_{6}+(8,8)_{0}+(6, \overline{6})_{2}+(\overline{6}, 6)_{-2}+ \\
+(3, \overline{3})_{2}+(\overline{3}, 3)_{-2}+(3, \overline{3})_{-4}+(\overline{3}, 3)_{4}
\end{gathered}
$$

where the subscripts denote the $\mathrm{U}(1)$ charges corresponding to the $Y_{U(1)}=\operatorname{diag}(1,1,1,-1,-1,-1)$ generator of $S U(6)$. After the 175 multiplet develops VEV in the $S U(3) \times S U(3) \times U(1)$ singlet - 
$(1,1)_{0}$ component, the $(3, \overline{3})_{2}+(\overline{3}, 3)_{-2}$ fragments are absorbed by appropriate gauge bosons and become unphysical.

Thus, the main feature of 175 IRREP is that it contains only the $G_{I}=S U(3)_{C} \times S U(3)_{W} \times U(1)$ singlet (among all maximal subgroups of $S U(6)$ ), and thus instead of multidegenerate vacua we have only one $S U(6)$-s breaking channel $(1, \mathrm{c}) . S U(3)_{C}$ is ordinary color group and $S U(3)_{W}$ contains weak $S U(2)_{W}$ group. If we introduce a pair of sextet-antisextet scalar superfields $H$ and $\bar{H}$, which develop VEVs on their sixth components, than $S U(3)_{W} \times U(1)$ will break to $S U(2)_{W} \times U(1)_{Y}$ group and doubletantidoublet pair from $H$ and $\bar{H}$, respectively will be absorbed. If in the theory there exists another pair of sextet-antisextet superfields $-H^{\prime}+\bar{H}^{\prime}$ and they are associated with $H+\bar{H}$ superfields by global symmetry, e.g. $S U(2)_{H}$, then one pair of doublets may remain massless after symmetry breaking. As we will see later discrete or continuous $R$ symmetry is sufficient to exclude some unacceptable terms from the superpotential.

Thus, we choose the scalar content of our model as $\Phi(175)+H_{m}(6)+\bar{H}^{m}(\overline{6})$, where $m$ is the $S U(2)_{H}$ index. The 175 breaks $S U(6)$ down to the $G_{I}$ subgroup, and the $6+\overline{6}$ fields break $S U(3)_{W} \times U(1)$ to $S U(2)_{W} \times U(1)_{Y}$. It is easy to check that the VEV structure of $\Phi(175)$ s singlet has the form:

$$
\begin{aligned}
\Phi_{124}^{124}=\Phi_{125}^{125}=\Phi_{126}^{126}=\Phi_{134}^{134}= & \Phi_{135}^{135}=\Phi_{136}^{136}=\Phi_{234}^{234}=\Phi_{235}^{235}=\Phi_{236}^{236}=-V \\
\Phi_{145}^{145}=\Phi_{146}^{146}=\Phi_{156}^{156}=\Phi_{245}^{245}= & \Phi_{246}^{246}=\Phi_{256}^{256}=\Phi_{345}^{345}=\Phi_{346}^{346}=\Phi_{356}^{356}=V \\
\Phi_{123}^{123}= & -\Phi_{456}^{456}=3 V
\end{aligned}
$$

where 1,2,3 and 4,5,6 respectively stand for the $S U(3)_{C}$ and $S U(3)_{W}$ indices, while for the VEVs of the sextet-antisextets we have:

$$
<H_{m}>=<\bar{H}^{m}>=\left(\begin{array}{cccccc}
0 & 0 & 0 & 0 & 0 & v \\
0 & 0 & 0 & 0 & 0 & 0
\end{array}\right)
$$

$V$ and $v$ can be found from the potential. From (ब) it is easy to see, that VEV of any odd power of $\Phi$ is zero:

$$
\operatorname{Tr}<\Phi^{2 \mathrm{~N}+1}>=0
$$

and consequently $\operatorname{Tr}<\Phi^{3}>=0$. In order to obtain nonzero $V$ and $v$, it is necessary to introduce also two gauge singlet superfields $s_{1}$ and $s_{2}$. Then the most general $S U(6) \times S U(2)_{H}$ invariant renormalizable superpotential

$$
W_{1}=\left(M_{\Phi}+\sigma_{1} s_{1}+\sigma_{2} s_{2}\right) \Phi^{2}+\left(M_{H}+h_{1} s_{1}+h_{2} s_{2}\right) \bar{H}^{m} H_{m}+\lambda \Phi^{3}+W_{1}\left(s_{1}, s_{2}\right)
$$

has the accidental global $S U(6)_{\Phi} \times U(6)_{H_{1}} \times U(6)_{H_{2}} \equiv S U(6)_{\Phi} \times U(6)_{H}^{2}$ symmetry under the independent rotation of $\Phi$ and $\bar{H}^{m}+H_{m}$ superfields. The symmetry of the superpotential is higher then the symmetry of the full Lagrangian. The VEV of $\Phi$ (eq. (4) breaks the $S U(6)_{\Phi}$ symmetry to $S U(3)_{\Phi} \times S U(3)_{\Phi} \times U(1)_{\Phi}$. 
Then $(3, \overline{3})_{2}+(\overline{3}, 3)_{-2}$ fragments from $\Phi(175)$ become goldstone modes. The VEVs of $H+\bar{H}$ (eq. (5)) break $U(6)_{H}^{2}$ to $U(5)_{H_{1}} \times U(6)_{H_{2}}$ and two pairs of $5+\overline{5}$ from $H_{m}+\bar{H}^{m}$ become massless Goldstones (The both pair remain massless due to $S U(2)_{H}$ symmetry). Thus due to the $S U(6)_{\Phi} \times U(6)_{H}^{2}$ global symmetry the color triplets are left massless along with the doublets components. In order to render the triplets massive, we have to avoid this global symmetry. The one way to do so is to include the higher order non-renormalizable terms in the superpotential.

In doing so, we observe that although the $d=5$ term $\bar{H}^{m} \Phi^{2} H_{m}$ violates the $S U(6)_{\Phi} \times U(6)_{H}^{2}$ symmetry, it does not lead to the desirable DT splitting. The reason is that the VEV of $\Phi^{2}$ can couple to $H, \bar{H}$ only in the $S U(6)$ singlet channel. In expanded form this term reads:

$$
\left(\Phi^{2}\right)_{D}^{C} \bar{H}^{D, m} H_{C, m}=\Phi_{A^{\prime} B^{\prime} C^{\prime}}^{A B C} \Phi_{A B D}^{A^{\prime} B^{\prime} C^{\prime}} \bar{H}^{D, m} H_{C, m}
$$

while from (四) we see that $\left\langle\Phi^{2}>_{D}^{C} \sim V^{2} \operatorname{diag}(1,1,1,1,1,1)_{D}^{C}\right.$. Therefore, this structure gives the same contribution to the mass terms of doublet and triplet fragments in $H, \bar{H}$, and if doublets remain massless, the mass of triplets also will vanish. Thus we have to include in the superpotential the $d=6$ term

$$
\bar{H}^{m} \Phi^{3} H_{m}
$$

One of the invariants (9) has the form:

$$
\left(\Phi^{3}\right)_{D}^{C} \bar{H}^{D, m} H_{C, m}=\Phi_{A^{\prime} B^{\prime} C^{\prime}}^{A B C} \Phi_{A_{1} B_{1} C_{1}}^{A^{\prime} B^{\prime} C^{\prime}} \Phi_{A B D}^{A_{1} B_{1} C_{1}} \bar{H}^{D, m} H_{C, m}
$$

Using (4) we see, that $\left\langle\Phi^{3}>_{D}^{C} \sim V^{3} \operatorname{diag}(1,1,1,-1,-1,-1)_{D}^{C}\right.$. Thus to obtain reasonable DT splitting it is necessary to include the (9) term in the superpotential.

Among the quartic terms there is $\alpha\left(\bar{H}^{m} H_{n}\right)\left(\bar{H}^{n} H_{m}\right)$ term, where brackets denote summation by $S U(6)$ indices. Expanding this term by $S U(2)_{H}$ indices we get:

$$
\left(\bar{H}^{m} H_{n}\right)\left(\bar{H}^{n} H_{m}\right)=\left(\bar{H}^{1} H_{1}\right)^{2}+2\left(\bar{H}^{1} H_{2}\right)\left(\bar{H}^{2} H_{1}\right)+\left(\bar{H}^{2} H_{2}\right)^{2}
$$

If only first pair develops VEV, then the doublets which come from this pair are goldstone bosons. First term from the (11) gives contribution to the mass of the first doublet-antidoublet pair, namely $2 \alpha v^{2}$. However the term (11) does not take part in the formation of the mass of the second doubletantidoublet pair and consequently the mass of the latter will be nonzero. If both pairs of scalar components from sextet-antisextet superfields have nonzero VEVs, there exists mixing between Higgs doublets. One eigenvalue of the mass matrix corresponds to the eigenstate which is a goldstone boson. Therefore nonlinear terms in $H$ (or $\bar{H}$ ) give the different contributions to the doublets' masses. In any case one doublet-antidoublet pair will be goldstone, but the second pair of doublets will have the undesirable mass (It is easy to verify, that another quartic term $\left(\bar{H}^{m} H_{m}\right)^{2}$ does not spoil the hierarchy). Therefore, we 
must exclude the term (11) by some symmetry reasons in such a way as to keep the term (9) and get the nonzero $V$ and $v$ in the limit of unbroken SUSY.

Below we present two models which satisfy these conditions and thus lead to the desirable DT splitting.

\section{Two Models}

Model 1. Let us introduce the discrete $R$-symmetry $Z_{3}$ under which all scalar superfields and the superpotential transform as

$$
\left(\Phi, \bar{H}^{m}, H_{m}\right) \rightarrow e^{i \frac{2 \pi}{3}}\left(\Phi, \bar{H}^{m}, H_{m}\right) ; \quad W \rightarrow e^{-i \frac{2 \pi}{3}} W
$$

Then the most general $S U(6) \times S U(2)_{H} \times Z_{3}$ invariant superpotential including the terms up to the fifth order has a form

$$
W=M_{H} \bar{H}^{m} H_{m}+a \bar{H}^{m} \Phi^{3} H_{m}+M_{\Phi} \Phi^{2}+b \Phi^{5}
$$

where under $a$ and $b$ terms all possible contractions by $S U(6)$ indices must be understood (we shall consider these terms in details later).

As it was mentioned above, the $\Phi(175)$ contains only $S U(3)_{C} \times S U(3)_{W} \times U(1)$ singlet with (1) vacuum structure. Thus it is impossible to change this direction by (5) structure because there does not exist another direction for 175 in the group space. The $F_{H_{A, m}}$ term has the form:

$$
F_{H_{A, m}}=M_{H} \bar{H}^{A, m}+a \bar{H}^{B, m}\left(\Phi^{3}\right)_{B}^{A}
$$

From $F_{H}=0$ condition with $\langle H>$ and $<\bar{H}>$ having the form (5), we find

$$
<\Phi^{3}>_{6}^{6}=-\frac{M_{H}}{a}
$$

and therefore

$$
<\Phi^{3}>_{B}^{A}=\frac{M_{H}}{a} \operatorname{diag}(1,1,1,-1,-1,-1)_{B}^{A}
$$

By substituting the $\Phi^{3}$ in (13) by (16), we can calculate the masses of the doublet and triplet components from the sextet-antisextet pairs. It is easy to see that for doublets $M_{D}=0$ and for triplets $M_{T}=2 M_{H}$.

As we see two pairs of doublet-antidoublets remain massless, while the two pairs of triplet-antitriplets have the masses of order $M_{H}$. One pair of doublet-antidoublet is absorbed by appropriate gauge fields and the second one survives after the symmetry breaking. There exists mixing between triplets (antitriplets) from 175 and triplets (antitriplets) from $H_{1}\left(\bar{H}^{1}\right)$. The mass matrix for the triplet (antitriplet) components has the following form: 


$$
\begin{aligned}
& 3_{175} \quad 3_{H_{1}} \quad 3_{H_{2}}
\end{aligned}
$$

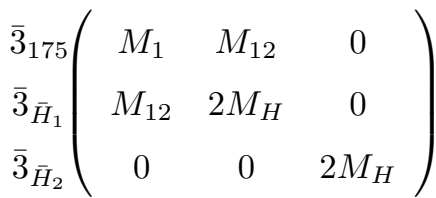

where

$$
\operatorname{det}\left(\begin{array}{cc}
M_{1} & M_{12} \\
M_{12} & 2 M_{H}
\end{array}\right)=0
$$

and thus one eigenvalue of the mass matrix is zero and the corresponding eigenstate is a goldstone boson. Other two nonzero eigenvalues are $2 M_{H}+M_{1}$ and $2 M_{H}$; their magnitudes must be not less than $10^{16} \mathrm{GeV}$ to avoid fast proton decay due to the $d=5$ operators. As we will see below, the $M_{H}$ can be compatible with a proton stability. To demonstrate this let us elaborate the superpotential in more details. After substituting $\Phi$ and $H+\bar{H}$ in (13) by (卧, (5) and using (6) all $b$ terms become zero. There exist seven possible $\bar{H} \Phi^{3} H$ invariants:

$$
\begin{aligned}
I_{1} & =\Phi_{A^{\prime} B^{\prime} C^{\prime}}^{A B C} \Phi_{A_{1} B_{1} C_{1}}^{A^{\prime} B^{\prime} C^{\prime}} \Phi_{A B D}^{A_{1} B_{1} C_{1}} \bar{H}^{D} H_{C} \\
I_{2} & =\Phi_{A_{1} B_{1} C_{1}}^{A B C} \Phi_{A B C^{\prime}}^{A_{1} A^{\prime} B^{\prime}} \Phi_{C A^{\prime} B^{\prime}}^{B_{1} C_{1} D} \bar{H}^{C^{\prime}} H_{D} \\
I_{3} & =\Phi_{A_{1} B_{1} C^{\prime}}^{A B C} \Phi_{A B C_{1}}^{A_{1} A^{\prime} B^{\prime}} \Phi_{C A^{\prime} B^{\prime}}^{B_{1} C_{1} D} \bar{H}^{C^{\prime}} H_{D} \\
I_{4} & =\Phi_{A^{\prime} B^{\prime} C^{\prime}}^{A B C} \Phi_{A A_{1} B_{1}}^{A^{\prime} B^{\prime} C^{\prime}} \Phi_{B C D}^{A_{1} B_{1} C_{1}} \bar{H}^{D} H_{C_{1}} \\
I_{5} & =\Phi_{A^{\prime} B^{\prime} C^{\prime}}^{A B C} \Phi_{A B A_{1}}^{A^{\prime} B^{\prime} B_{1}} \Phi_{C B_{1} C_{1}}^{C^{\prime} A_{1} D} \bar{H}^{C_{1}} H_{D} \\
I_{6} & =\Phi_{A^{\prime} B^{\prime} C^{\prime}}^{A B C} \Phi_{A_{1} B_{1} C_{1}}^{A^{\prime} B^{\prime} C^{\prime}} \Phi_{A B C}^{A_{1} B_{1} C_{1}} \bar{H}^{D} H_{D} \\
I_{7} & =\Phi_{A^{\prime} B^{\prime} C^{\prime}}^{A B C} \Phi_{A B A_{1}}^{A^{\prime} B_{1} C_{1}} \Phi_{C B_{1} C_{1}}^{B^{\prime} C^{\prime} A_{1}} \bar{H}^{D} H_{D}
\end{aligned}
$$

and thus

$$
a \bar{H}^{m} \Phi^{3} H_{m} \equiv \sum_{i=1}^{7} a_{i} I_{i}
$$

where $a_{i}$ are the parameters of dimension $\mathrm{GeV}^{-2}: a_{i}=\frac{\lambda_{i}}{M^{2}}$ where $\lambda_{i}$ are dimensionless parameters and $M$ is some cutoff mass parameter. Using (19), (20) we get:

$$
W(V, v)=M_{H} v^{2}-48\left(36 \lambda_{1}+2 \lambda_{2}+\lambda_{3}+24 \lambda_{4}+8 \lambda_{5}\right) \frac{V^{3} v^{2}}{M^{2}}+36^{2} M_{\Phi} V^{2}
$$

The numbers are the combinator factors. From the condition $F_{V}=F_{v}=0$ we have:

$$
\begin{aligned}
V & =M\left(\frac{M_{H}}{M}\right)^{1 / 3}\left[48\left(36 \lambda_{1}+2 \lambda_{2}+\lambda_{3}+24 \lambda_{4}+8 \lambda_{5}\right)\right]^{-1 / 3} \\
v & =6 M\left(\frac{6 M_{\Phi}^{3}}{M^{2} M_{H}}\right)^{1 / 6}\left(36 \lambda_{1}+2 \lambda_{2}+\lambda_{3}+24 \lambda_{4}+8 \lambda_{5}\right)^{-1 / 3}
\end{aligned}
$$

For $M \sim 10^{17} \mathrm{GeV}$ (superstring scale), $M_{H} \sim 10^{16} \mathrm{GeV}, M_{\Phi} \sim 10^{15} \mathrm{GeV}, \lambda_{i} \sim 10^{-1}$ we get $V \sim v \sim$ $10^{16} \mathrm{GeV}$. Consequently, the respective $\mathrm{d}=5$ operator is suppressed by the $M_{H} \sim 10^{16} \mathrm{GeV}$ scale. 
We have demonstrated how the cancelation of doublets' masses occurs if the superpotential has the form (13). The higher terms, which are permitted by the $S U(6) \times S U(2)_{H} \times Z_{3}$ symmetry and contain unacceptable term (11) are:

$$
\frac{1}{M_{P l}^{5}} \Phi^{4} \times(\bar{H})^{2} \times(H)^{2}, \quad \frac{1}{M_{P l}^{5}} \Phi^{2} \times(\bar{H})^{3} \times(H)^{3}, \quad \frac{1}{M_{P l}^{5}}(\bar{H})^{4} \times(H)^{4}
$$

where all possible contractions by $S U(6)$ and $S U(2)_{H}$ indices is assumed. The doublet's mass induced from these terms will have the magnitude $M_{D} \sim M_{G}\left(\frac{M_{G}}{M_{P l}}\right)^{5}$ and for $M_{P l} \sim 10^{19} \mathrm{GeV}$, we have $M_{D} \sim$ $10-100 \mathrm{GeV}$, which is indeed the desirable value for the " $\mu$-term".

One may ask why the cut-off parameters $M$ and $M_{P l}$ respectively in (21) and (23) have different magnitudes? Let us assume that term (9) is obtained by the heavy particle exchange mechanism [11]. Let us introduce the pairs of vector supermultiplets: $\overline{210}^{m}+210_{m}\left(m\right.$ is $S U(2)_{H}$ index $) .210 \equiv \Psi_{A B C}^{A^{\prime} B^{\prime}}$ is antisymmetric with respect to up and down indices and $\sum \Psi_{A B C}^{A B^{\prime}}=0$. If the superpotential for $\bar{\Psi}^{m}+\Psi_{m}$ has the form:

$$
W_{\Psi}=M \bar{\Psi}^{m} \Psi_{m}+\alpha_{1} \Phi \bar{\Psi}^{m} H_{m}+\alpha_{2} \Phi \Psi_{m} \bar{H}^{m}+\alpha_{3} \Phi \bar{\Psi}^{m} \Psi_{m}
$$

the lowest operators which are obtained after integrating out the heavy $\bar{\Psi}^{m}+\Psi_{m}$ fields are $\frac{1}{M} \bar{H}^{m} \Phi^{2} H_{m}$ and $\frac{1}{M^{2}} \bar{H}^{m} \Phi^{3} H_{m}$. It is easy to check that the higher operators containing the combination (11) will not be induced by exchanges of $\Psi+\bar{\Psi}$. The terms of eq. (23) containing the multiplier (11) may be induced only by the nonperturbative gravity effects and thus they will be suppressed by the Planck scale.

Note, that $W_{\Psi}$ do not has the definite $Z_{3}$ charge as $W$ (see eq. (12)). To improve this drawback let us introduce the gauge singlet superfield $-s$ and instead of $Z_{3}$ symmetry $Z_{8}$, under which the scalar superfields and the superpotential transform as:

$$
\begin{gathered}
\left(\bar{H}^{m}, H_{m}, \bar{\Psi}^{m}, \Psi_{m}\right) \rightarrow e^{i \frac{2 \pi}{8}}\left(\bar{H}^{m}, H_{m}, \bar{\Psi}^{m}, \Psi_{m}\right), \quad \Phi \rightarrow \Phi, \\
s \rightarrow e^{i \frac{2 \pi}{4}} s ; \quad W \rightarrow e^{i \frac{2 \pi}{4}} W
\end{gathered}
$$

were $W=W_{1}+W_{\Psi} ; W_{\Psi}$ is given by (24) and

$$
W_{1}=M_{H} \bar{H}^{m} H_{m}+a \bar{H}^{m} \Phi^{3} H_{m}+\alpha s \Phi^{2}+\frac{\beta}{M_{P l}^{2}} s^{5}
$$

the lowest term, which contains multiplear (11) and permitted by these symmetries is: $\frac{1}{M_{P l}^{4}} \bar{H}^{2} \times H^{2} s^{3}$ from (26) it is easy to verify that if $\frac{\alpha}{\beta} \sim 10^{-4}$ then $\left\langle s>\sim 10^{16} \mathrm{GeV}\right.$ and we have $M_{D} \sim 10 \mathrm{TeV}$.

Model 2. The second possibility to restrict all nonlinear terms in $H$ (or $\bar{H}$ ), which in general may spoil the hierarchy, is the continuous $R$-symmetry. For example if $R_{H}=1, R_{\Phi}=R_{\bar{H}}=0$ (in the units of the W-charge) then the superpotential will have the form:

$$
W=\bar{H}^{m}\left(M_{H}+a_{1} \Phi^{2}+a_{2} \Phi^{3}+\ldots\right) H_{m}
$$


Note that if $R_{\Psi}=R_{H}$ and $R_{\bar{\Psi}}=0$ then the terms in $W_{\Psi}$ (see eq. (24) ) have the same $R$ charge as $W$ (eq. (27)), and thus they also can be incorporated in the theory. From the condition $F_{H}=0$ the VEV of $\Phi$ is fixed. It is possible to satisfy the $F_{\Phi}=0$ condition in such a way as to get nonzero VEV-s for $H(\bar{H})$, if we arrange their VEV-s as it was suggested in ref. [10]:

$$
<H_{1}>=<\bar{H}^{2}>=(0,0,0,0,0, v) ; \quad<H_{2}>=<\bar{H}^{1}>=0
$$

It is obvious, that $F_{\Phi}=0$ condition is satisfied and $v$ is undetermined. In other words, (27) has an $F$-flat and $D$-flat vacuum.

\section{Conclusions}

We have considered the SUSY $S U(6)$ theory in which the GUT symmetry breaking occurs due to the Higgs superfield in the 175 representation. It does not contain the Higgs doublet fragments and can break the $S U(6)$ symmetry only in the $S U(3)_{C} \times S U(3)_{W} \times U(1)$ channel. By introducing also two pairs of the scalar superfields in $6+\overline{6}$ representation which are related by the custodial global $S U(2)_{H}$ symmetry, we have constructed two models in which the DT-splitting occurs naturally. In these models the discrete or continuous $R$ symmetries are used for obtaining the desired structure of superpotential, which along with the renormalizable terms also include all allowed nonrenormalizable ones. It is worth to stress that the latter also play a crucial in providing the large masses to the Higgs triplets while for the doublet components they may induce the desirable value for " $\mu$-term".

The fermion sector of the model may be constructed in the same manner as in ref. [10], if we have one $\overline{6}^{m}+15\left(m\right.$ is $S U(2)_{H}$ index) anomaly-free fermion supermultiplet per generation. The Yukawa superpotential which generates up and down fermion masses has a form:

$$
W_{Y}=g_{d} \overline{6}^{m} 15 \bar{H}^{n} \epsilon_{m n}+\frac{g_{u}}{M_{0}} 1515 H_{m} H_{n} \epsilon_{m n}
$$

which implies the following assignment of charges: $\overline{6}^{m} \rightarrow e^{i \frac{2 \pi}{3}} \overline{6}^{m}$ in case of $Z_{3}$ symmetry and $\overline{6}^{m} \rightarrow$ $e^{i \frac{2 \pi}{8}} \overline{6}^{m}$ in case of $Z_{8}$ for the Model 1 , and $R_{15}=-R_{H} / 2$ and $R_{\overline{6}^{n}}=3 R_{H} / 2$ for the Model 2. The last term in (29) may be obtained by exchange of the heavy superfields with mass of order $M_{0}$ [11]. For the details concerning the fermion sector we refer to [10, where this question is discussed.

Let us conclude with the following comment: Neglecting the threshold effects we will have the standard unification of the three gauge couplings. Taking into account the threshold effects due to the $75+50+\overline{50}$ multiplets, the picture will be changed. It was shown in [12 that nonminimal (missing multiplet) $S U(5)$ model with these IRREPs allows small values of the SUSY breaking scale $m_{\text {susy }}$ for any $\alpha_{s}\left(m_{Z}\right)$ in the experimentally allowed range. In the context of our model this result cannot be directly applied due to the different gauge sector. The detailed study of the problem of the gauge coupling unification in our model and its implications for the proton decay is the subject of a separate investigation. 


\section{Acknowledgements}

We thank J.Chkareuli and G.Dvali for useful discussions. This work was supported in part by the ISF grant No.MXL000 and Georgian Government and ISF grant No.MXL200.

Z.T. thanks G.Senjanovic and especially Z.Berezhiani for useful discussions, also the International Atomic Energy Agency and UNESCO for hospitality at the International Center for Theoretical Physics, Trieste.

\section{References}

[1] S.Dimopoulos and H.Georgi, Nucl.Phys. B193 (1981) 150;

N. Sakai, Z.Phys. C11 (1981) 153; E. Witten, Nucl.Phys. B188 (1981) 573.

[2] E.Witten, Phys.Lett. B105 (1981) 267;

D.V.Nanopoulos and Tamvakis, Phys.Lett. B113 (1982) 151;

L.Ibanez and G.G.Ross, Phys.Lett. B110 (1982) 215.

[3] J.Polchinski and L.Susskind, Phys.Rev. D26 (1982) 3661;

H.P.Nilles, M.Srednicki and D.Wyler, Phys.Lett. B124 (1983) 337; A.B.Lahanas, ibid. 341;

L.Alvarez-Gaume, J.Polchinski and M.B.Wise, Nucl.Phys. B221 (1983) 495;

D.Nemeschansky, Nucl.Phys. B234 (1984) 379.

[4] S.Dimopoulos and F.Wilczek, in Erice Summer Lectures, Plenum, New York, 1981;

H.Georgi, Phys.Lett. B108 (1982) 283; B.Grinstein, Nucl.Phys. B206 (1982) 387;

A.Masiero, D.V.Nanopoulos, K.Tamvakis and T.Yanagida, Phys.Lett. B115 (1982) 380;

for recent analysis, see J.L.Lopez and D.V.Nanopoulos, hep-ph/9508253.

[5] S.Dimopoulous and F.Wilczek, NSF-ITP-82-07 (unpublished);

M.Srednicki, Nucl.Phys. B202 (1982) 327; K.S.Babu and S.M.Barr, Phys.Rev. D48 (1993) 5354.

[6] K.S.Babu and S.M.Barr, Phys.Rev. D50 (1994) 3529.

[7] K.Inoue, A.Kakuto and T.Takano, Progr. Theor. Phys. 75 (1986) 664;

A.Anselm and A.Johansen, Phys.Lett. B200 (1988) 331.

[8] Z.Berezhiani and G.Dvali, Sov. Phys. Lebedev Institute Reports 5 (1989) 55;

R.Barbieri, G.Dvali and A.Strumia, Nucl.Phys. B391 (1993) 487;

R.Barbieri, G.Dvali, A.Strumia, Z.Berezhiani, L.Hall, Nucl.Phys. B432 (1994) 49;

Z.Berezhiani, Phys.Lett. B355 (1995) 481.

[9] Z.Berezhiani, C.Csaki, L.Randall, Nucl.Phys. B444 (1995) 61. 
[10] G.Dvali, Phys.Lett. B324 (1994) 59.

[11] C.D.Froggatt and H.B.Nielsen, Nucl.Phys. B147 (1979) 277;

Z.G.Berezhiani, Phys.Lett. B129 (1983) 99; B150 (1985) 177;

S.Dimopoulos, Phys.Lett. B129 (1983) 417.

[12] Y.Yamada, Z.Phys. C60, (1993) 83. 